\title{
The return of the membrane paradigm? Black holes and strings in the water tap
}

\author{
Vitor Cardoso 12
}

Department of Physics and Astronomy, The University of Mississippi, University, MS 38677-1848, USA

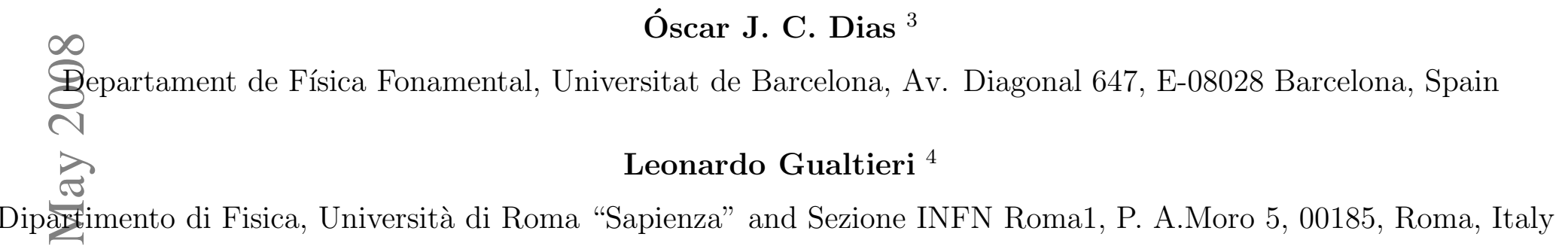

\begin{abstract}
Several general arguments indicate that the event horizon behaves as a stretched membrane. We propose using this relation to understand gravity and dynamics of black objects in higher dimensions. We provide evidence that (i) the gravitational Gregory-Laflamme instability has a classical counterpart in the Rayleigh-Plateau instability of fluids. Each known feature of the gravitational instability can be accounted for in the fluid model. These features include threshold mode, dispersion relation, time evolution and critical dimension of certain phase transitions. Thus, we argue that black strings break in much the same way as water from a faucet breaks up into small droplets. (ii) General rotating black holes can also be understood with this analogy. In particular, instability and bifurcation diagrams for black objects can easily be inferred. This correspondence can and should be used as a guiding tool to understand and explore physics of gravity in higher dimensions.
\end{abstract}

\footnotetext{
${ }^{1}$ email: vcardoso@phy.olemiss.edu

${ }^{2}$ Also at Centro de Física Computacional, Universidade de Coimbra, P-3004-516 Coimbra, Portugal

3 email: odias@ub.edu

${ }^{4}$ email: gualtieri@roma1.infn.it
} 


\section{Introduction}

Gravity in higher dimensions has a long and tortuous story, beginning with the first attempts by Kaluza and Klein to unify gravity and electromagnetism. Recent proposals include large extra-dimensional models and braneworlds, advocated as a possible solution to the hierarchy problem of gauge couplings. Perhaps the most famous example of higher dimensional theories is string theory, in which the previous scenarios can be embedded. Higher-dimensional theories generally possess more degrees of freedom, thus providing a richer arena to describe physical phenomena. However, this extended freedom has a cost: the parameter space to be searched is much wider and calculations in higher dimensional gravity are technically very challenging. Thus a case-by-case search has to be performed, with the associated time-costly computations. Moreover, it is not uncommon that the necessary mathematical machinery is not even available, making the problem altogether impossible to handle.

It is thus of the utmost importance to sidestep the unwanted complex technicalities and infer or identify directly the general, physically important features of the problem. A very popular approach to bypass hard technical computations is to use analogue models [1, 2, 3]: one builds on previously established, well understood results, usually concerning a completely different setup, to infer the general behavior of the problem at hand. The power of drawing analogies is that they enable one to predict and gain intuition.

What we propose here is to use fluids held together by surface tension as models for gravitational objects in a general number of spacetime dimensions [4, 5]. In simple terms, this means that some of the effects seen for instance in water or soap bubbles should have a natural counterpart in the gravitational sector. The idea of using analogues of this kind to extract useful information is not new: more than 200 years ago Plateau [2] conducted many experiments on liquid drops. The purpose of such studies was to model giant liquid masses held together by self-gravitation (planets and stars) using centimeter-sized drops held together by surface tension. Another well-known case, where the fluid drop model proved extremely useful, is Bohr and Wheeler's [1] description of nuclear fission as the rupture of a charged liquid drop, where now the surface tension plays the role of nuclear forces. A final widely known example, closely related to this essay, is the "membrane paradigm" for black holes by Thorne et al [3]. This paradigm establishes a rigorous mapping between the properties of the event horizon and those of a stretched membrane, endowing the former with well-defined mechanical and electromagnetic properties. 
There is an extra motivation to relate the properties of event horizons to fluids with surface tension. Take the first law of black hole mechanics [6] describing how a (for simplicity, uncharged, static) black hole, characterized by its mass or energy $E$ and horizon area $A$, evolves when we throw an infinitesimal amount of matter into it:

$$
d E=T d A
$$

This law is nothing but a manifestation of the thermodynamical properties of black holes: they can be ascribed a surface gravity proportional to its temperature $T_{\text {Hawking }}=4 T=1 /(8 \pi E)$. Alternatively, as first argued by Smarr [7], Eq. (11) can be interpreted as a law for fluids, with $T$ being an effective surface tension [8]. This is rather intuitive: in fluids the potential energy is associated with the storage of energy at the surface, therefore proportional to the area. The statement that, for a given energy, the black object prefers the configuration with more entropy translates to the well-known hydrodynamic property that, for a given volume, the fluid picks the configuration with less surface area.

Our purpose here is to elaborate on the relationship between event horizons and membranes in higher dimensional arenas. We show that with this correspondence at hand, many of the properties of black objects in higher dimensions can be derived with little effort. It is also an extremely powerful tool to predict new phenomena and in general to allow one to understand gravity in higher dimensions.

\section{Rayleigh-Plateau and Gregory-Laflamme instabilities}

An illuminating example of the power of this analogy concerns black strings and branes [9]. These are extended black holes: the horizon, instead of having the topology of a sphere, can have for instance the topology of sphere times a line - a cylinder. The simplest black string is described by the metric of a $D$-dimensional Schwarzschild black hole times a line. Black strings are unstable against gravity, in a mechanism known as Gregory-Laflamme (GL) instability [10]. The GL mechanism makes any small perturbation with wavelength $\lambda$ of the order of, or larger than, the radius of the cylinder $R_{0}$ grow exponentially with time. For wavelengths larger than

a threshold $\lambda_{c}, k_{c} R_{0} \equiv 2 \pi R_{0} / \lambda_{c} \sim \sqrt{D}$ (for large $D$ ), the instability appears [11]. In this essay, we propose the dual object to the black string to be a fluid cylinder held by surface tension. As shown by Plateau [2], a cylinder longer than its circumference is energetically unstable to breakup: any axisymmetric small disturbance decreases the surface area of the cylinder. This 
is known as the Rayleigh-Plateau (RP) instability. Indeed, consider a disturbed hyper-cylinder, with $D-1$ spatial directions, radius $R_{0}$ and a transverse direction $z$. A simple calculation [4]

yields $A=A_{0}\left(1+\frac{\epsilon^{2} R_{1}^{2}}{4 R_{0}^{2}}\left[k^{2} R_{0}^{2}-(D-2)\right]\right)$ for the area of the disturbed cylinder, with $A_{0}$ the undisturbed area. The potential energy per unit length is therefore $P \propto\left[k^{2} R_{0}^{2}-(D-2)\right] T$. We conclude that the system is unstable for $k_{c} R_{0}<\sqrt{D-2}$, since in this case the perturbation decreases the potential energy. Note the remarkable quantitative agreement between the threshold modes of the RP and GL instabilities for large number of spatial dimensions $D$.

The agreement holds for all known features of these instabilities [4]. The dispersion relation (mode frequency vs wavenumber) of the two instabilities are quite similar and have the same dependency on space dimension $D$ [4]. Non-axisymmetric modes are stable both in the RP and GL instabilities. The existence of a critical dimension for both objects provides an extra non-trivial check on the duality we are proposing: one finds that, for $D \leq D_{\mathrm{c}}$ (with $D_{\mathrm{c}}=11$ ), a spherical configuration has less surface area than the cylinder and is thus a favored endpoint [4]. For $D>D_{\text {c }}$ this is no longer true. A similar critical dimension is present in the GL side [11]: for $D<D_{\mathrm{c}}\left(D_{\mathrm{c}}=13\right)$ the black string is entropically unstable against the formation of a spherical black hole. If rotation is added to the fluid, the strength of the fluid instability increases due to centrifugal force effects. Thus, the proposed duality predicts that even extremal black strings should be unstable. This was very recently confirmed in the gravitational sector [12].

\section{The fate of black strings and their duals}

The full time evolution of the RP instability is well known (numerically and experimentally [13]) while so far, only the initial stage of the GL has been numerically studied [11]. The available results are consistent with the duality viewpoint: starting from a single sinusoidal perturbation both develop an almost cylindrical thread or neck in between the two half rounded boundary regions. These agreements raise of course several questions. The first is related to the endpoint of the instability. What can we say about it, what insights can the analogue or effective model offer? The RP instability makes a cylinder pinch off. We end up with an array of main and satellite drops with different sizes with less surface area than the initial cylinder [2, 13]. The analogy then suggests that a similar configuration should exist as a static solution on the gravity side. Very recently, this configuration of multi-black holes with different masses was constructed perturbatively as a solution of Einstein gravity [14]. In analogy with the fluid, we can understand this gravitational endpoint as due to the fact that the array of black holes has more entropy than the initial uniform black string. 


\section{A critical dimension for phase transitions of black strings and fluids}

The threshold mode in the GL analysis signals a bifurcation to a new static branch of nonuniform strings [15]. A study by Sorkin [11] concluded that for $D<D_{*}$ (with $D_{*} \sim 12,13$ ) the phase transition from uniform to non-uniform black strings is of first order, while for larger $D$ it is second order, therefore smoother. Within the fluid dual there is a similar static branch and critical dimension $D_{*}$ for the phase transition. An heuristic, back-of-the-envelope computation can be done as follows. Take a fluid cylinder, and expand the geometry around the zero mode (presented above) of a static uniform cylinder. Specifically, consider the parametrization $r(z)=R_{0}+\epsilon R_{1} \cos \left(k_{c} z\right)+\epsilon^{2} R_{2}+\epsilon^{3} R_{3} \cos (3 K z)$, where we set $K=k_{c}+\epsilon k_{1}$. The factors $R_{2}$ and $k_{c}$ were determined in [4] by requiring volume conservation and area extremization: $R_{2}=-\frac{D-2}{4 R_{0}} R_{1}^{2}$ and $R_{0} k_{c}=\sqrt{D-2}$. Going to next order $\left(\epsilon^{3}\right)$, and demanding that both the uniform and the non-uniform cylinder have the same volume, we find $R_{3}=\frac{(D-1)(D-2)^{3 / 2}(D-4)}{64 k_{1} R_{0}^{4}} R_{1}^{4}$, $k_{1}$ is left undetermined, and

$$
\frac{A_{\text {non-uniform }}-A_{\text {uniform }}}{A_{\text {uniform }}}=\frac{(D-2)(D-4+\sqrt{10})(D-4-\sqrt{10})}{64 R_{0}^{4}} \epsilon^{4} R_{1}^{4} .
$$

We conclude that at $D=D_{*} \sim 7.2$ there is a change in the smoothness of the phase transition; in this case the transition seems to be smoother for $D<D_{*}$. It would be very interesting to perform a more rigorous analysis of this transition, within a mathematically rigorous framework.

\section{Rotation and bifurcation: black holes and black rings}

The correspondence proposed here can improve - and strengthen - our understanding of other gravitational objects in higher dimensions. Take for instance spherical black holes. Their fluid analogues - liquid drops - are solutions of fluid dynamics in higher dimensions. The evolution of these objects once rotation is added was reported in [5]. The spherical drop (for zero rotation) acquires a spheroidal shape as the rotation increases. For rotation larger than a certain critical value this axisymmetric configuration is unstable and a two-lobed configuration forms. This suggests that highly rotating black holes are unstable. In four dimensions, the Kerr bound is small enough to avoid the development of such instabilities. However, in dimensions higher than six, rotating Myers-Perry black holes [16] have no Kerr-like bound, and the instability might well set in. Recent arguments by Emparan and Myers [17], using gravitational physics arguments, lend further support to this claim. For rotations larger than the above mentioned critical value, the axisymmetric configuration is unstable, but it still is an equilibrium solution. 
It was further shown in [5] that for even higher rotation rates the axisymmetric family eventually goes over to a torus or ring-like configuration. This has the natural gravitational counterpart in the black ring solution [18. An obvious question is whether or not the black ring solution is stable. Computations in the dual fluid model are straightforward: these solutions are unstable.

\section{Where do we go from here?}

There is solid evidence that the correspondence surface gravity $\rightleftarrows$ surface tension allows one to study a plethora of phenomena related to gravitational physics in higher dimensions. The wish list for a successful completion of this programme includes: (i) a proof of the correspondence. One such proof exists for black holes in four dimensions, but the analog membrane is rather unusual, with negative bulk viscosity and a non-vanishing shear viscosity. The examples worked out so far hint at something slightly more surprising: as one climbs up in spacetime dimension the analogy we described in this essay seems to work better and better, and thus it seems like surface tension is the only ingredient that needs to be considered. (ii) Confirmation of the predictions of the dual fluid model. Some of these, namely rotation effects and the existence of multi-black holes, have already been confirmed, but progress in the gravitational sector is slow. The ultimate goal is of course to use the dual model as a guiding tool, to go hand in hand with its gravitational cousin. That is precisely the usefulness of analogies.

\section{Acknowledgments}

We would like to thank the many suggestions by the participants of the KITP Program "Scanning New Horizons: GR Beyond 4 Dimensions", of the "16th Midwest Relativity Meeting", and of the Hebrew University programme: "Einstein's Gravity in Higher Dimensions". In particular, we warmly thank Emanuele Berti, Luca Bombelli, Marco Cavaglià, Roberto Emparan, Barak Kol, Troels Harmark, Jordan Hovdebo, Akihiro Ishibashi, Donald Marolf, Robert Myers, Niels Obers, Leonard Parker, Michael Seifert and Toby Wiseman for valuable comments and suggestions, that eventually lead to a solid picture of how this correspondence works. This work was partially funded by Fundação para a Ciência e Tecnologia (FCT) - Portugal through projects PTDC/FIS/64175/2006 and POCI/FP/81915/2007. OD acknowledges financial support provided by the European Community through the Intra-European Marie Curie contract MEIF-CT-2006-038924. 


\section{References}

[1] N. Bohr and J. A. Wheeler, "The mechanism of nuclear fission," Phys. Rev. 56, 426 (1939).

[2] J. Plateau, Statique expérimentale et théorique des liquides soumis aux seules forces moléculaires, (Paris, Gauthier-Villars, 1873).

[3] K. S. Thorne, D. A. MacDonald and R. H. Price, Black Holes: The Membrane Paradigm (Yale University Press, 1986); see also M. Parikh and F. Wilczek, "An action for black hole membranes," Phys. Rev. D 58, 064011 (1998).

[4] V. Cardoso and O. J. C. Dias, "Rayleigh-Plateau and Gregory-Laflamme instabilities of black strings," Phys. Rev. Lett. 96, 181601 (2006).

[5] V. Cardoso and L. Gualtieri, "Equilibrium configurations of fluids and their stability in higher dimensions," Class. Quant. Grav. 23, 7151 (2006).

[6] J. M. Bardeen, B. Carter and S. W. Hawking, "The four laws of black hole mechanics," Commun. Math. Phys. 31, 161 (1973).

[7] L. Smarr, "Mass formula for Kerr black holes," Phys. Rev. Lett. 30, 71 (1973).

[8] R. Defay, I. Prigogine, A. Bellemans and D. H. Everett, Surface tension and absorption, (John Wiley and Sons, New York, 1966).

[9] G. T. Horowitz and A. Strominger, "Black strings and p-branes," Nucl. Phys. B 360, 197 (1991).

[10] R. Gregory and R. Laflamme, "Black strings and p-branes are unstable," Phys. Rev. Lett. 70, 2837 (1993).

[11] T. Harmark, V. Niarchos and N. A. Obers, "Instabilities of black strings and branes," Class. Quant. Grav. 24 (2007) R1; B. Kol, "The phase transition between caged black holes and black strings: A review," Phys. Rept. 422 (2006) 119.

[12] B. Kleihaus, J. Kunz and E. Radu, "Rotating nonuniform black string solutions," JHEP 0705, 058 (2007)

[13] J. Eggers, "Nonlinear dynamics and breakup of free surface flows," Rev. Mod. Phys. 69, 865 (1997).

[14] O. J. C. Dias, T. Harmark, R. C. Myers and N. A. Obers, "Multi-black hole configurations on the cylinder," Phys. Rev. D 76 (2007) 104025.

[15] S. S. Gubser, "On non-uniform black branes," Class. Quant. Grav. 19, 4825 (2002); E. Sorkin, "A critical dimension in the black-string phase transition," Phys. Rev. Lett. 93, 031601 (2004).

[16] R. C. Myers and M. J. Perry, "Black holes in higher dimensional space-times," Annals Phys. 172, 304 (1986).

[17] R. Emparan and R. C. Myers, "Instability of ultra-spinning black holes," JHEP 0309, 025 (2003). 
[18] R. Emparan and H. S. Reall, "A rotating black ring in five dimensions," Phys. Rev. Lett. 88, 101101 (2002). 